\title{
Multiphoton Optical Heterodyne Detection
}

\author{
MALVIN CARL TEICH, SENIOR MEMBER, IEEE
}

\begin{abstract}
After reviewing recent work on multiphoton direct detection and single-photon heterodyne detection, we examine the behavior of a multiphoton absorption heterodyne receiver. Expressions are obtained for the detector response, signal-to-noise ratio (SNR), and minimum detectable power for a number of cases of interest. Receiver performance is found to depend on the higher order correlation functions of the radiation field and on the local oscillator (LO) irradiance. Although performance similar to that of the single-photon heterodyne receiver can theoretically be achieved, practical problems would appear to make this difficult. A physical interpretation of the process in terms of the absorption of monochromatic and nonmonochromatic photons is given. The double-quantum case is treated in particular detail; the results of a preliminary experiment are presented and suggestions for future experiments are provided.
\end{abstract}

\section{INTRODUCTION}

I NASMUCH as single-quantum optical heterodyne detection and multiple-quantum optical direct detection are by now well-understood processes, it seems natural to investigate the properties of a detection scheme in which both are performed simultaneously. In this paper, we obtain the response and the signal-to-noise power ratio for a multiple-quantum absorption heterodyne receiver, with particular attention devoted to the simplest case, i.e., the mixing of two waves in a double-quantum infrared or optical device.

After briefly considering the relevant results pertinent to each of the processes individually (Section I), we derive the combination device response for the general multiple-quantum photomixing process in Section II. In Section III, the response for the important two-quantum case is obtained and given a physical interpretation. In Section IV, we obtain the signal-tonoise ratio (SNR) for a receiver using a multiphoton optical heterodyne device, and compare it with the SNR for conventional optical heterodyne detection. The results of a twophoton experiment are presented in Section $V$, while a suggested setup for future experiments, as well as the applicability of the scheme in general, is reserved for Section VI.

\section{A. Multiphoton Direct Detection}

The ordinary photoeffect was discovered by Hertz in 1887 and explained in terms of the absorption of a single quantum of light by Einstein in his now famous work published in 1905 [1]. It was not until 1959, however, that the relationship between the statistics of an arbitrary incident radiation field and the emitted photoelectrons was firmly established by Mandel [2]. Consideration of the general photodetection process in terms of quantum-electrodynamic co-

Manuscript received January 27, 1975. This work was supported in part by the National Science Foundation, and in part by the John Simon Guggenheim Memorial Foundation.

The author is with the Department of Electrical Engineering and Computer Science, Columbia University, New York, N.Y. 10027, and is a Fellow of the John Simon Guggenheim Memorial Foundation, New York, N.Y. herent states of the radiation field was undertaken by Glauber [3] in 1963, and by Kelley and Kleiner [4] in 1964, and provides a convenient starting point for calculations involving multiple-photon as well as single-photon absorptions.

Multiple-quantum photoemission, being a higher order effect, is most easily observed in the absence of ordinary (firstorder) photoemission. For the two-quantum case, it becomes important when

$$
\frac{1}{2} e \phi<h \nu<e \phi
$$

where $h \nu$ is the photon energy of the incident radiation and $e \phi$ is the work function of the material under consideration. (Even when (1) is satisfied, however, it should be kept in mind that small amounts of single-quantum photoemission can arise from excited electrons in the Fermi tail [5].) The two-quantum photoeffect was first experimentally observed in 1964. Using a GaAs laser, Teich et al. [6] observed the effect in sodium metal, while Sonnenberg et al. [7] induced it in $\mathrm{Cs}_{3} \mathrm{Sb}$ with a Nd-doped glass laser. Since that time, there have been a number of experimental measurements of second- and higher order photoelectric yields in a variety of materials [8]-[12] .

Theoretical work has focused on two aspects of the problem: perturbation theory and other calculations of the transition probabilities in the material, and the effect on the transition probability of the statistical nature of the radiation field. Makinson and Buckingham [13] were the first to predict the second-order effect and calculate its magnitude based on a surface model of photoemission; this work was expanded by Smith [14], Bowers [15], and Adawi [16]. The analogous volume calculation was performed by Bloch [17] and later corrected by Teich [8] , and Teich and Wolga [9] .

All of the models predict a two-quantum dc photocurrent $W_{\mathrm{dc}}^{(2)}$ (expressed in amperes) proportional to the square of the incident radiation power $P$ and inversely proportional to the irradiated area $A$. Using the results of a number of authors [8], [9], [14]-[17], we can therefore write the doublequantum de photocurrent as

$$
W_{\mathrm{dc}}^{(2)}=\Lambda^{(2)}(\lambda, T) P \propto I P .
$$

Here $\Lambda^{(2)}$ is the two-quantum yield expressed in amperes per watt [9], $\lambda$ is the radiation wavelength, $T$ is the sample temperature, $P$ is the radiation power expressed in watts, and $I$ is the irradiance at the detector expressed in watts per square centimeter. The two-quantum efficiency (electrons/photon) is denoted by $\eta_{2}$, and is related to the two-quantum yield by the relationship

$$
\Lambda^{(2)}=(e / h v) \eta_{2} \propto I .
$$

Here, the quantity $(h \nu / e)$ is the incident photon energy expressed in electronvolts and is of order unity. For the $k$-photon process, defining $W_{\mathrm{dc}}^{(k)}, \Lambda^{(k)}$, and $\eta_{k}$ as the $k$-photon analogs of the quantities defined above, the following generalized results 
are obtained:

$$
W_{\mathrm{dc}}^{(k)}=\Lambda^{(k)}(\lambda, T) P \propto I^{k-1} P
$$

and

$$
\Lambda^{(k)}=(e / h \nu) \eta_{k} \propto I^{k-1} .
$$

Typical numerical values for the two-quantum yield are [9] $\Lambda_{\mathrm{Na}}^{(2)}(8450 \AA, 300 \mathrm{~K}) \sim 8 \times 10^{-16} I$ and $\Lambda_{\mathrm{Cs} 3 \mathrm{Sb}}^{(2)}(10600 \AA$, $300 \mathrm{~K}) \sim 5 \times 10^{-11} I \mathrm{~A} / \mathrm{W}$. Again $I$ represents the irradiance at the detector in watts per square centimeter. These values, even when precisely measured, can vary by a factor (usually $\leqslant 2$ ) depending on the coherence properties of the inducing radiation, as we now consider.

Theoretical work relating to multiple-quantum statistical effects began in 1966 with an examination of the higher order field correlation functions by Teich and Wolga [18] and by Lambropoulos et al. [19]. This was followed by more detailed calculations by Mollow [20] and by Agarwal [21]. All of these studies predicted a factor of $k$ ! enhancement for the magnitude of certain $k$-quantum processes induced by chaotic (rather than coherent) sources. This enhancement was later observed in the two-quantum photoeffect by Shiga and Imamura [10], and in second-harmonic generation (SHG) by Teich et al. [22]. The theoretical relationship between twoquantum photocurrent spectra and the incident radiation statistics was then obtained by Diament and Teich [23], and compared with the analogous single-quantum results previously given by Freed and Haus [24]. In 1969 two-quantum photocounting distributions were calculated for amplitude-stabilized, chaotic, and generalized laser sources by Teich and Diament [25]. This work was extended to higher order photocounting distributions by Barashev in 1970 [26], who also wrote an excellent and comprehensive review article on multiple-quantum photoemission and photostatistics in 1972 [27].

\section{B. Single-Photon Heterodyne Detection}

Being a first-order effect, ordinary optical and infrared heterodyne detection has received far more attention than has multiple-quantum detection. The usefulness of the technique in applications ranging from spectroscopy to radiometry has been well documented. The effect was first observed by Forrester et al. [28] in a classic experiment using two Zeeman components of a visible (incoherent) spectral line. With the development of the laser, photomixing became relatively easy to observe and was studied by Javan et al. [29] at $1.15 \mu \mathrm{m}$ using a He-Ne laser, and by Siegman et al. [30b)] at $6943 \AA$ with a ruby laser. Extending this work into the middle infrared, Teich et al. used a $\mathrm{CO}_{2}$ laser at $10.6 \mu \mathrm{m}$ in conjunction with a copper-doped germanium photoconductive detector operated at $4 \mathrm{~K}$ [31], and with a lead-tin selenide photovoltaic detector operated at $77 \mathrm{~K}$ [32].

The observed signal-to-noise power ratio for these experiments was found to behave in accordance with the theoretical expression obtained for parallel plane-polarized beams under ideal conditions [31]-[34], i.e.,

$$
\mathrm{SNR}^{(1)}=\eta_{1} P_{1} / h \nu \Delta f \text {. }
$$

Here, $\eta_{1}$ is the detector quantum efficiency (electrons per photon), $P_{1}$ is the received signal radiation power, $h v$ is the photon energy, and $\Delta f$ is the receiver bandwidth. For radiation beams which are not parallel to within an angle $\theta=\lambda / d$, with $d$ the detector aperture, the SNR is reduced below the value given in (6) by spatial averaging of the mixing signal over the detector aperture. This effect was studied in detail by Siegman and by Mandel and Wolf [34], and is frequently referred to as "wash-boarding." Similar calculations have been effected for focused radiation beams, as first considered by Read and Fried [35].

Shortly thereafter, this work was extended to experimental and theoretical studies of the statistical nature of the heterodyne signal resulting from the beating of a coherent wave with a Gaussian scattered wave [36], [37]. Although the stochastic nature of this signal depends in detail on the irradiance statistics, the SNR is found to be essentially independent of the higher order correlation functions of the field [36], [37]. Furthermore, using the first-order coherent field results of Titulaer and Glauber [38] for absorption detectors, an explicit calculation for the case of two-beam photomixing has shown that sum- and double-frequency components do not appear in the detected current, and that the heterodyne process can be interpreted in terms of the annihilation of a single (nonmonochromatic) photon [39], [40], as was qualitatively appreciated by Forrester et al. [28]. Finally, a concise review of the basic theoretical and experimental aspects of heterodyne detection in the infrared and optical, as well as a partial review of the literature, was prepared by Teich in 1970 [41]. Another such review, emphasizing nonlinear heterodyne detection, will appear shortly [42].

\section{Multiphoton Photomixing}

We begin this section by considering a two-quantum absorption detector initially in the ground state. The detector response $W^{(2)}$ at the space-time point $x_{a}=\vec{r}_{a}, t_{a}$ may be written in terms of the second-order correlation function $G^{(2)}$ [3], [18], [20], [25], and is given by

$$
\begin{aligned}
W^{(2)} & \propto \operatorname{tr}\left\{\rho E^{-}\left(x_{a}\right) E^{-}\left(x_{a}\right) E^{+}\left(x_{a}\right) E^{+}\left(x_{a}\right)\right\} \\
& \equiv G^{(2)}\left(x_{a} x_{a} x_{a} x_{a}\right) .
\end{aligned}
$$

Here, $\rho$ is the density operator for the field, and $E^{-}$and $E^{+}$ represent the negative- and positive-frequency portions of the electric field operator $E$, respectively. We assume that the final state of the detector is much broader than the bandwidth of the incident radiation, and that a broad band of final states is accessible [20], [21].

If we specifically consider the mixing of two single-mode, amplitude-stabilized, first-order coherent waves, both of which are well collimated, parallel, plane polarized along a common unit vector, and normally incident onto a photosensitive material, we may write the positive portion of the electric field operator $E^{+}$as the superposition of two scalar fields

$$
E^{+}=\varepsilon_{1}^{0} e^{-i \omega_{1} t}+\varepsilon_{2}^{0} e^{-i \omega_{2} t}
$$

with angular frequencies $\omega_{1}$ and $\omega_{2}$. This is equivalent to assuming a semiclassical approach which makes use of the analy tic signal [43]. The complex wave amplitude $\mathscr{E}_{i}^{0}$ can be expressed in terms of its absolute magnitude $\left|\mathscr{E}_{i}^{0}\right|$ and a phase 
factor $e^{i \alpha_{i}}$ such that

$$
\begin{aligned}
& \mathscr{E}_{1}^{0}=\left|\mathscr{E}_{1}^{0}\right| e^{i \alpha} \\
& \mathscr{E}_{2}^{0}=\left|\mathscr{E}_{2}^{0}\right| e^{i \beta} .
\end{aligned}
$$

Under these conditions, the quantum-statistical detector responses can be written in terms of the fields as

$$
\begin{aligned}
& \operatorname{tr}\left\{\rho E^{-} E^{+}\right\} \Rightarrow\left|\varepsilon_{1}^{0}\right|^{2}+\left|\varepsilon_{2}^{0}\right|^{2} \\
& \quad+2\left|\&_{1}^{0}\right|\left|\varepsilon_{2}^{0}\right| \cos \left[\left(\omega_{1}-\omega_{2}\right) t+(\beta-\alpha)\right]
\end{aligned}
$$

and

$$
\begin{aligned}
& \operatorname{tr}\left\{\rho E^{-} E^{-} E^{+} E^{+}\right\} \Rightarrow\left(\left|\mathscr{E}_{1}^{0}\right|^{2}+\left|\mathscr{E}_{2}^{0}\right|^{2}\right. \\
& \left.\quad+2\left|\mathscr{E}_{1}^{0}\right|\left|\mathscr{E}_{2}^{0}\right| \cos \left[\left(\omega_{1}-\omega_{2}\right) t+(\beta-\alpha)\right]\right)^{2} .
\end{aligned}
$$

These expressions are scalar quantities and contain no spatial dependence because of the assumptions of plane polarization, parallel beams, and normal incidence.

Generalizing these results to sinusoidal beam photomixing in which the $k$-photon detector response is the normally ordered product [3]

$$
W^{(k)} \propto \operatorname{tr}\left\{\rho\left[E^{-}\left(x_{a}\right)\right]^{k}\left[E^{+}\left(x_{a}\right)\right]^{k}\right\}
$$

and using the binomial theorem leads to a heterodyne signal given by

$$
\begin{aligned}
& W^{(k)}=\zeta_{k}\left(\left\{\left|\mathbb{E}_{1}^{0}\right|^{2}+\left|\varepsilon_{2}^{0}\right|^{2}\right\}^{k}\right. \\
& +\left(\begin{array}{c}
k \\
1
\end{array}\right)\left\{\left|\Theta_{1}^{0}\right|^{2}+\left|\varepsilon_{2}^{0}\right|^{2}\right\}^{k-1}\left\{2\left|\&_{1}^{0}\right|\left|\varepsilon_{2}^{0}\right|\right. \\
& \left.\cdot \cos \left[\left(\omega_{1}-\omega_{2}\right) t+(\beta-\alpha)\right]\right\} \\
& +\left(\begin{array}{l}
k \\
2
\end{array}\right)\left\{\left|\varepsilon_{1}^{0}\right|^{2}+\left|\varepsilon_{2}^{0}\right|^{2}\right\}^{k-2}\left\{2\left|\varepsilon_{1}^{0}\right|\left|\varepsilon_{2}^{0}\right|\right. \\
& \left.\cdot \cos \left[\left(\omega_{1}-\omega_{2}\right) t+(\beta-\alpha)\right]\right\}^{2}+\cdots \\
& +\left(\begin{array}{c}
k \\
r
\end{array}\right)\left\{\left|\mathscr{E}_{1}^{0}\right|^{2}+\left|\mathscr{E}_{2}^{0}\right|^{2}\right\}^{k-r}\left\{2\left|\mathscr{E}_{1}^{0}\right|\left|\mathscr{E}_{2}^{0}\right|\right. \\
& \left.\cdot \cos \left[\left(\omega_{1}-\omega_{2}\right) t+(\beta-\alpha)\right]\right\}^{r}+\cdots \\
& \left.+\left\{2\left|\mathscr{E}_{1}^{0}\right|\left|\mathscr{E}_{2}^{0}\right| \cos \left[\left(\omega_{1}-\omega_{2}\right) t+(\beta-\alpha)\right]\right\}^{k}\right) \text {. }
\end{aligned}
$$

Here $\zeta_{k}$ represents a proportionality constant for the $k$-photon process. The leading dc terms are proportional to $\left|\&_{1}^{0}\right|^{2 k}$ and $\left|\varepsilon_{2}^{0}\right|^{2 k}$, and may be associated with the absorption of $k$ monochromatic photons, each of which arises from a given beam ( 1 and 2, respectively). The highest frequency current component is proportional to

$$
\left|\varepsilon_{1}^{0}\right|^{k}\left|\varepsilon_{2}^{0}\right|^{k} \cos \left[k\left(\omega_{1}-\omega_{2}\right) t+\phi\right]
$$

and corresponds to the absorption of $k$ nonmonochromatic photons, each of which must be associated with both of the beams. It is evident from the above that multiple- and sumfrequency terms do not appear in the $k$-photon absorption heterodyne detector output, in analogy with the result for the one-quantum case [39]-[41] .

\section{Two-Photon Photomixing}

Inserting the constants $\zeta$ for the one- and two-quantum cases in (13), the detector responses for coherent signal mixing are, respectively,

$$
\begin{aligned}
W^{(1)}= & \zeta_{1}\left(\left|\mathscr{\varepsilon}_{1}^{0}\right|^{2}+\left|\mathscr{\varepsilon}_{2}^{0}\right|^{2}\right. \\
& \left.+2\left|\varepsilon_{1}^{0}\right|\left|\varepsilon_{2}^{0}\right| \cos \left[\left(\omega_{1}-\omega_{2}\right) t+(\beta-\alpha)\right]\right)
\end{aligned}
$$

and

$$
\begin{aligned}
W^{(2)}= & \xi_{2}\left(\left|\&_{1}^{0}\right|^{4}+\left|\&_{2}^{0}\right|^{4}+2\left|\mathscr{E}_{1}^{0}\right|^{2}\left|\mathscr{E}_{2}^{0}\right|^{2}\right. \\
& +4\left|\mathscr{E}_{1}^{0}\right|^{3}\left|\mathscr{E}_{2}^{0}\right| \cos \left[\left(\omega_{1}-\omega_{2}\right) t+(\beta-\alpha)\right] \\
& +4\left|\mathscr{E}_{1}^{0}\right|\left|\mathscr{E}_{2}^{0}\right|^{3} \cos \left[\left(\omega_{1}-\omega_{2}\right) t+(\beta-\alpha)\right] \\
& \left.+4\left|\&_{1}^{0}\right|^{2}\left|\&_{2}^{0}\right|^{2} \cos ^{2}\left[\left(\omega_{1}-\omega_{2}\right) t+(\beta-\alpha)\right]\right) .
\end{aligned}
$$

Using the double-angle formula for the last term in (15), $W^{(2)}$ may also be written as

$$
\begin{aligned}
W^{(2)}= & \zeta_{2}\left(\left|\varepsilon_{1}^{0}\right|^{4}+\left|\varepsilon_{2}^{0}\right|^{4}+4\left|\varepsilon_{1}^{0}\right|^{2}\left|\varepsilon_{2}^{0}\right|^{2}\right. \\
& +4\left|\varepsilon_{1}^{0}\right|^{3}\left|\varepsilon_{2}^{0}\right| \cos \left[\left(\omega_{1}-\omega_{2}\right) t+(\beta-\alpha)\right] \\
& +4\left|\varepsilon_{1}^{0}\right|\left|\varepsilon_{2}^{0}\right|^{3} \cos \left[\left(\omega_{1}-\omega_{2}\right) t+(\beta-\alpha)\right] \\
& \left.+2\left|\varepsilon_{1}^{0}\right|^{2}\left|\varepsilon_{2}^{0}\right|^{2} \cos \left[2\left(\omega_{1}-\omega_{2}\right) t+2(\beta-\alpha)\right]\right)(16)
\end{aligned}
$$

when this $\cos ^{2}$ term is present. As noted previously, doubleand sum-frequency terms are absent.

It is not difficult to associate various second-order correlation functions $G^{(2)}\left(x_{a} x_{b} x_{c} x_{d}\right) \equiv[a b c d]$ with (15). (When two beams are present, we must consider a space-time point for each of the beams so that the index in $G^{(2)}$ takes on two values [18].) Thus the first term, $\left|\&_{1}^{0}\right|^{4}$, may be associated with [1111], the second with [2222], the third with [1221] and [2112], the fourth with the four permutations of [1112], the fifth with the four permutations of [2221], and the sixth with the four permutations of [1212], with $b \neq c$. The coefficient of each term in (15) is therefore equal to the number of permutations in the appropriate form of the correlation function for that term. The physical interpretation follows immediately: the first two dc terms in (15) arise from the absorption of two monochromatic photons, both from the same beam. The third dc term, which exists in two permutations with $b=c$, arises from the two ways in which two single monochromatic photons can be absorbed, one from each beam. The fourth and fifth terms correspond to the absorption of a single monochromatic photon from one of the beams plus a single nonmonochromatic photon which must be associated with both beams. These terms therefore contribute currents at the difference frequency $\left(\omega_{1}-\omega_{2}\right)$, in analogy with the single-quantum heterodyne interference term [39]-[41]. The final term corresponds to the absorption of two nonmonochromatic photons, and therefore varies at double the difference frequency, i.e., at $2\left(\omega_{1}-\omega_{2}\right)$; clearly there is no analogous process possible in the one-quantum case.

We note that the absorption of two nonmonochromatic photons imparts an additional dc value to the double-differencefrequency term, as may be seen by comparing (15) and (16). This additional term, of magnitude $2\left|\varepsilon_{1}^{0}\right|^{2}\left|\varepsilon_{2}^{0}\right|^{2}$, appears in the presence of double-quantum photomixing; in the absence of such photomixing, we must obtain $W^{(2)}$ from (15) and not from (16). In this latter case, the detector response reduces to the previously obtained result [18]

$$
\begin{aligned}
W^{(2)}[\text { mixing absent }] & =\xi_{2}\left(\left|\&_{1}^{0}\right|^{4}+\left|\&_{2}^{0}\right|^{4}+2\left|\&_{1}^{0}\right|^{2}\left|\&_{2}^{0}\right|^{2}\right) \\
& =\xi_{2}^{\prime}\left(I_{1}+I_{2}\right)^{2}
\end{aligned}
$$

where $I_{i}$ represents the intensity of the $i$ th beam and $\zeta_{2}^{\prime}$ is a new proportionality constant. 
The results presented above can be expanded to modulated, noncoherent, and nonparallel beam mixing. As an example, we consider two ideal amplitude-stabilized nonparallel $(\theta>\lambda / d)$ plane traveling waves impinging on a two-quantum detector, so that washboarding can occur. In contrast to the one-quantum case, the detector responds to the square of the spatial intensity variation across the detector aperture at every instant of time, resulting in a factor of 2 enhancement in the $\mathrm{dc}$ cross term, as obtained with pure temporal mixing. Thus the two-quantum dc photocurrent will in general be enhanced due to spatiotemporal intensity variations (interference fringes); the magnitude of this enchancement depends on the system configuration. Experimental evidence for two-quantum enhancement due to spatial variations has, in fact, been provided by Shiga and Imamura [10] and by Teich et al. [22] .

As a final example, we consider mixing due to radiation which is nonsinusoidal (i.e., not coherent to all orders). We

\section{Signal-To-Noise Ratio}

We now follow the usual procedure used for the singlequantum case [31]-[33], [37] to calculate the approximate SNR for $k$-photon sinusoidal heterodyne detection. We begin with two-quantum photomixing, neglecting the doubledifference-frequency component and assuming that the ac signal is at the fundamental-difference-frequency (IF) between the two waves. Thus, considering the mixing of two parallel coherent waves as described in Section II, (16) yields

$$
\begin{aligned}
W_{\mathrm{IF}}^{(2)}= & 4 \zeta_{2}\left(\left|\mathscr{E}_{1}^{0}\right|^{3}\left|\varepsilon_{2}^{0}\right|+\left|\mathscr{E}_{1}^{0}\right|\left|\varepsilon_{2}^{0}\right|^{3}\right) \\
& \cdot \cos \left[\left(\omega_{1}-\omega_{2}\right) t+(\beta-\alpha)\right] .
\end{aligned}
$$

and

$$
W_{\mathrm{dc}}^{(2)}=\zeta_{2}\left(\left|\varepsilon_{1}^{0}\right|^{4}+\left|\varepsilon_{2}^{0}\right|^{4}+4\left|\&_{1}^{0}\right|^{2}\left|\&_{2}^{0}\right|^{2}\right)
$$

so that

$$
W^{(2)}=\left\{1+\frac{4\left(\left|\mathscr{E}_{1}^{0}\right|^{3}\left|\mathscr{E}_{2}^{0}\right|+\left|\mathscr{E}_{1}^{0}\right|\left|\mathscr{E}_{2}^{0}\right|^{3}\right) \cos \left[\left(\omega_{1}-\omega_{2}\right) t+(\beta-\alpha)\right]}{\left|\mathscr{E}_{1}^{0}\right|^{4}+\left|\mathscr{E}_{2}^{0}\right|^{4}+4\left|\mathscr{E}_{1}^{0}\right|^{2}\left|\mathscr{E}_{2}^{0}\right|^{2}}\right\} W_{\mathrm{dc}}^{(2)}
$$

consider two parallel, plane-polarized, normally incident superimposed beams of radiation from the same chaotic source, one of which is a time-delayed version of the other (delay $\tau_{\delta}$ ) entering one double-quantum detector. This was previously shown to be equivalent to a self-integrating Hanbury-Brown-Twiss device [18]. For a thermal source in the absence of a beat signal, we find

$$
W^{(2)}[\text { mixing absent }]=2 \zeta_{2}^{\prime}\left(I_{1}^{2}+2 I_{1} I_{2}+I_{2}^{2}\right), \quad \tau_{\delta}<\tau_{c}
$$

and

$$
W^{(2)}[\text { mixing absent }]=2 \zeta_{2}^{\prime}\left(I_{1}^{2}+I_{1} I_{2}+I_{2}^{2}\right), \quad \tau_{\delta}>\tau_{c}
$$

where $\tau_{c}$ is the coherence time of the source. For $\tau_{\delta}<\tau_{c}$, (18) represents the enhancement of both the single-beam and the mixed-beam counting rates, arising from the tendency of these photons to arrive in correlated pairs (assuming that the detector intermediate state lifetime $\tau_{l} \ll<\tau_{c}$ ). For $\tau_{\delta}>\tau_{c}$, however, there is no correlation between the arrival time of a photon from one beam and the arrival time of a photon from the other. Thus the absorption of two photons from a single beam is enhanced by a factor of 2 relative to the absorption of one photon from each beam, leading to a cross term of 1 . This can also be qualitatively understood from the point of view of additive Gaussian fields; the sum of two fully correlated Gaussian random processes $\left(\tau_{\delta}<\tau_{c}\right)$ has a greater variance than that of two independent Gaussian random processes $\left(\tau_{\delta}>\tau_{c}\right)$ leading to an enhanced value for the cross term when $\tau_{\delta}<\tau_{c}$.

From the foregoing, it is clear that the double-quantum current can be calculated for a variety of configurations involving different relative time scales, angular separations, polarization properties, and statistical characteristics. Some additional examples are treated in [18]. Clearly, the second-order correlation functions of the field play an important role in determining the magnitude of the signal, in distinction to the one-quantum case.
We now assume that one of the waves (which we call the local oscillator or LO) is strong, i.e., $E_{2} \gg E_{1}$, in which case

$$
W_{\mathrm{IF}}^{(2)} \simeq 4\left(\left|\varepsilon_{1}^{0}\right| /\left|\varepsilon_{2}^{0}\right|\right) W_{\mathrm{dc}}^{(2)} \cos \left[\left(\omega_{1}-\omega_{2}\right) t+(\beta-\alpha)\right]
$$

and

$$
\left\langle\left[W_{\mathrm{IF}}^{(2)}\right]^{2}\right\rangle \simeq 8\left(\left|\varepsilon_{1}^{0}\right|^{2} /\left|\varepsilon_{2}^{0}\right|^{2}\right)\left[W_{\mathrm{dc}}^{(2)}\right]^{2} .
$$

The noise power can be obtained from the two-quantum photocurrent spectrum [23] which, in turn, is related to the stochastic nature of the radiation source. For a coherent and strong LO, however, the $k$-quantum counting statistics will be Poisson [25], [26], and the two-quantum (shot) noise power is then

$$
\left\langle\left[W_{n}^{(2)}\right]^{2}\right\rangle=2 e\left[W_{\mathrm{dc}}^{(2)}\right] \Delta f .
$$

Thus using (2), (24), and (25), the two-quantum SNR can be written as

$$
\mathrm{SNR}^{(2)} \simeq \frac{4 P_{1}}{e \Delta f}\left(\frac{W_{\mathrm{dc}}^{(2)}}{P_{2}}\right)=\frac{4 P_{1}}{e \Delta f} \Lambda^{(2)} .
$$

Using the relationship between the two-quantum yield $\Lambda^{(2)}$ and the two-quantum efficiency $\eta_{2}$ given in (3), we finally obtain

$$
\mathrm{SNR}^{(2)} \simeq 4 \eta_{2} P_{1} / h \nu \Delta f
$$

We recall from (3) that $\eta_{2}$ is itself proportional to the irradiance of the LO, and we must have $4 \eta_{2}<1$. The result is therefore similar to that for the single-quantum heterodyne detector given in (6); in that case, however, $\eta_{1}$ is independent of the LO. The two-quantum minimum detectable power (MDP) [31], [32] therefore becomes

$$
\operatorname{MDP}^{(2)} \simeq h \nu \Delta f / 4 \eta_{2} \text {. }
$$

This corresponds to a minimum number of photons $\pi_{\mathrm{min}}^{(2)}$ detectable in the resolution time of the receiver $\left[\tau_{r} \sim(\Delta f)^{-1}\right]$, 
given by

$$
\pi_{\min }^{(2)} \simeq\left(4 \eta_{2}\right)^{-1} \text {. }
$$

We note that SNR at the double-difference frequency, corresponding to the absorption of two nonmonochromatic photons, is reduced by the factor $\left(P_{1} / P_{2}\right)$. Clearly, using methods similar to those presented above and in [18] and [37], we can obtain analogous SNR expressions for photomixing with nonsinusoidal beams.

The SNR at the fundamental-difference frequency $\left(\omega_{1}-\omega_{2}\right)$ may also be obtained for coherent beam mixing in the $k$-photon absorption heterodyne detector. Following a series of steps similar to those given above, we find

$$
\begin{aligned}
& \operatorname{SNR}^{(k)} \simeq c_{k} \eta_{k} p_{1} / h \nu \Delta f \\
& \operatorname{MDP}^{(k)} \simeq h \nu \Delta f / c_{k} \eta_{k}
\end{aligned}
$$

and

$$
r_{\min }^{(k)} \simeq\left(c_{k} \eta_{k}\right)^{-1}
$$

where $c_{k}$ is a constant (dependent on $k$ ), and $\eta_{k}$ is proportional to $I^{k-1}$, where $I$ is the LO irradiance [see (5)]. Here $c_{k} \eta_{k}<1$.

\section{EXPERIMENT}

In this section we describe a preliminary set of experiments in which double-quantum photoemission was observed from a sodium surface simultaneously illuminated by two superimposed beams of laser radiation. While ac photomixing terms were not observed in these experiments, the measurements are consistent with the theoretical calculations given in Section III.

\section{A. Configuration}

The apparatus used for the experimental measurement of two-quantum photomixing is shown in the block diagram of Fig. 1. The radiation source was a pulsed GaAs multimode semiconductor injection laser operated at $77 \mathrm{~K}$ and emitting a peak radiation power of $400 \mathrm{~mW}$ at about $8450 \AA$. Mode shifts due to laser heating occurred during the pulse duration,

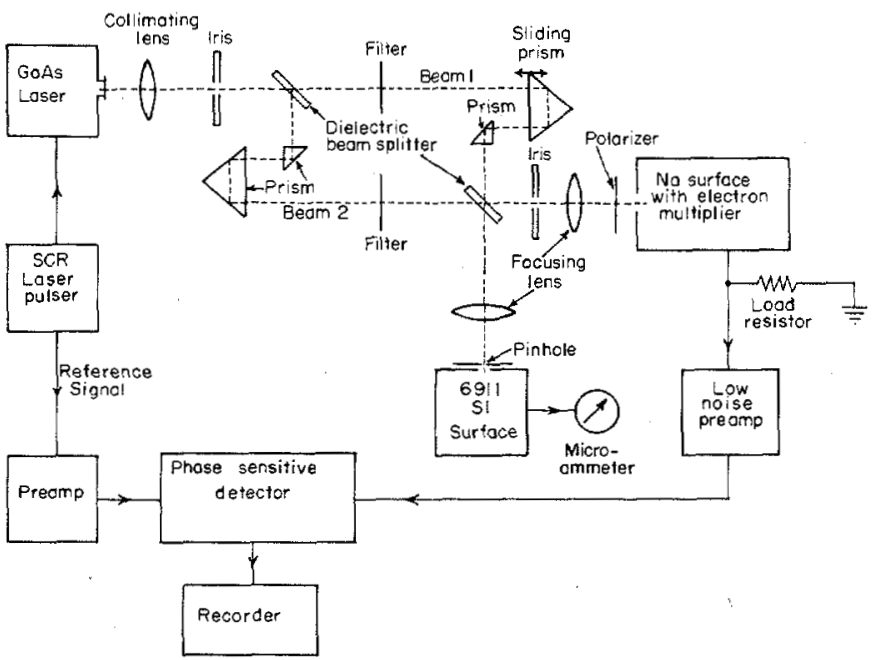

Fig. 1. Block diagram of the double-quantum photomixing experimental arrangement. which was about $35 \mu \mathrm{s}$. The radiation was collimated by a $10-\mathrm{cm}$ focal length lens, passed through an iris, and then through a configuration of dielectric beam splitters and antireflection coated prisms resembling a Mach-Zender interferometer. The beam splitters were approximately $\frac{2}{3}$ transmitting and were flat only to about 1 wavelength; the optical phase across the beam could therefore be considered to vary. The purpose of the interferometer configuration was to allow the irradiance of each beam (denoted as 1 and 2) to be independently controlled by means of calibrated attenuating filters. Beam 1 could also be time-delayed with respect to beam 2 by means of a sliding prism (see Fig. 1), but this capability was not important in these experiments where $\tau_{\delta}$ was always greater than $\tau_{c}$ due to the very small value of $\tau_{c}$. After passing through a second iris and a (6-cm focal length) focusing lens, the radiation was allowed to impinge on a specially constructed $\mathrm{Na}$-surface photomultiplier tube, which has been described previously [6], [8], [9]. A Polaroid type HN-7 sheet polarizer was aimost always placed at the front face of the photomultiplier as shown in Fig. 1 (the one exception will be noted later). The electron-multiplied current was passed through a 1-M $\Omega$ load resistor which fed a Princeton Applied Research (PAR) low-noise preamplifier followed by a PAR lock-in amplifier. Phase-sensitive detection was performed at $2.2 \mathrm{kHz}$, which is the fundamental repetition frequency of the pulsed laser output. Large integration times were used so that only the dc or average value of this current component was measured. The reference signal for the lock-in amplifier was obtained directly from the silicon-controlled-rectifier power supply [44] used to drive the laser.

Radiation from the other leg of the interferometer was focused onto a $25-\mu \mathrm{m}$ diameter pinhole which acted as an aperture stop at the face of a standard Dumont 6911 type S-1 photomultiplier. This provided a relatively accurate method for superimposing the two beams [8]. This is critical since the double-quantum response is inversely proportional to the illuminated area $A$. The beams were adjusted to achieve maximum output from the 6911 photomultiplier tube, a procedure which was often difficult and required a great deal of care.

\section{B. Method}

The following procedure was used in making a measurement. 1) The beams were aligned to provide maximum current from the 6911 photomultiplier. 2) Beam 1 was blocked and the double-quantum current $\overline{W_{2}^{(2)}}$ from beam 2 was maximized by imaging the laser junction on the sodium surface, and then recorded. Using a calibrated attenuating filter, it was ascertained that pure two-quantum emission was occurring, i.e., that $\overline{W_{2}^{(2)}} \propto I_{2}^{2}$, where $I_{2}$ represents the irradiance of beam 2 . 3) Beam 2 was blocked and the double-quantum current from beam 1 was recorded, after verifying that it was $\propto I_{1}^{2}$. (The constant of proportionality was taken to be the same in both cases.) 4) Both beams were then unblocked and, after once again verifying that pure double-quantum emission was occurring, the total average double-quantum current $\overline{W^{(2)}}$ (at the fundamental repetition frequency of $2.2 \mathrm{kHz}$ ) was recorded.

Experiments were performed with different value of $I_{1} / I_{2}$, 
obtained by attenuating one of the beams relative to the other by means of thin gelatin (Kodak Wratten) filters. Ordinary glass filters could not be used to provide the decrements of light intensity because refraction in the glass caused the imaged spot size and position to change thus altering the twoquantum current in an unpredictable way.

\section{Results}

The total average fundamental-repetition-frequency twoquantum photoelectric current $\overline{W^{(2)}}$, for different intensity ratios of the two constituent beams $\left(I_{1} / I_{2}\right)$, is presented in Fig. 2. The solid curve represents the equation $W^{(2)} \propto$ $\left(I_{1}+I_{2}\right)^{2}$ which is simply the parabola $\left(1+I_{1} / I_{2}\right)^{2}$ when the intensity $I_{2}$ is normalized to unity. This represents a cross term of $2 I_{1} I_{2}$, and is so labeled. The cross term of $4 I_{1} I_{2}$, on the other hand, is shown by the dashed line in Fig. 2. Only the highest observed values of $\overline{W^{(2)}}$ are plotted in Fig. 2, many more points having been found to lie below the curves. This has been attributed to the difficulty in obtaining precise alignment of the two radiation beams, and therefore superposition of the focused spots on the sodium surface.

The triangles in Fig. 2 represent data for linearly or partially polarized radiation, while the circle is for cross-polarized radiation (this is the one exception mentioned previously). The experimental measurements are consistent with the following interpretation. The laser output consists of a number of more-or-less independent Fabry-Perot modes which are changing during the pulsewidth due to heating of the laser junction [8]. The radiation may therefore be considered to behave as a Gaussian source with a coherence time $\tau_{c} \sim$ $(\Delta \nu)^{-1} \sim 10^{-13}$ s. Since the intermediate-state lifetime for the double-quantum sodium photodetector is much shorter

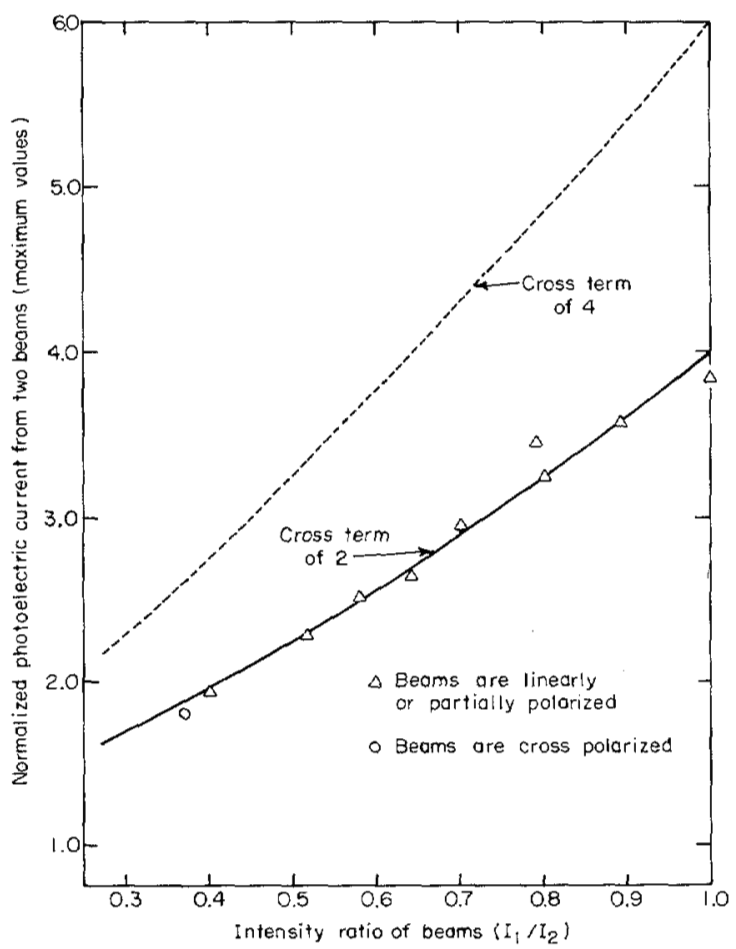

Fig. 2. The average double-quantum photocurrent from two beams (maximum values) versus the intensity ratio of the beams. than the radiation coherence time, the irradiance fluctuations result in a factor of 2 enhancement of the singlebeam photocurrents. As far as the irradiance cross term is concerned, the two-quantum current will also be enhanced by a factor of 2 owing to the random spatial irradiance fluctuations across the detector for the superimposed beams. Thus we obtain a relative cross terms of 2 , i.e., $\overline{W^{(2)}} \propto I_{1}^{2}+2 I_{1} I_{2}+$ $I_{2}^{2}$, in agreement with the data.

\section{Discussion}

From the foregoing, it is clear that multiple-quantum heterodyne detection is somewhat more complex than the analogous single-quantum process. In particular, the average detector response and the SNR are found to depend on the higher order correlation functions of the radiation field and on the LO irradiance. From a physical point of view, it has been possible to associate various terms in the detected current with specific kinds of photon absorptions. Calculations of the SNR and MDP for a number of cases have been carried out. The results of a preliminary two-quantum photomixing experiment are in agreement with the theory.

Although it appears that the $k$-photon heterodyne detector can be made to perform as well as the single-photon heterodyne detector by simply increasing the LO intensity, a number of practical problems would likely make this difficult. Inasmuch as the transition probabilities decrease rapidly as $k$ is increased, and are furthermore proportional to $A^{1-k}$, it appears that very high LO intensities would be required to place $\eta_{k}$ anywhere in the vicinity of 0.1 for $k>2$. Aside from alignment problems, these high intensities could result in thermionic emission from cathode heating, or possibly cathode damage.

The two-quantum case is therefore likely to be the most interesting, and also the easiest to examine experimentally. A possible arrangement for studying the effect in a more detailed and controlled fashion is the following. The radiation from a $0.5-\mathrm{mW} \mathrm{He}-\mathrm{Ne}$ laser, operating at a wavelength of $1.15 \mu \mathrm{m}$, is passed through an acoustooptic modulator (which splits it into two frequencies) and a focusing lens. A $5-\mu \mathrm{m}$ focused spot size, corresponding to an area of $2.5 \times 10^{-7} \mathrm{~cm}^{2}$, would then provide an incident irradiance $I \sim 2 \times 10^{3} \mathrm{~W} / \mathrm{cm}^{2}$. Using a $\mathrm{Cs}_{3} \mathrm{Sb}$ photocathode with a work function $\sim 2.05 \mathrm{eV}$ and a yield [7] $\sim 5 \times 10^{-11} I \mathrm{~A} / \mathrm{W}$, a two-quantum current $\sim 5 \times 10^{-11}$ A may then be obtained. An experiment of this nature would allow the validity of (15), (16), and (27) to be examined. A YAG:Nd laser could be substituted for the $\mathrm{He}-\mathrm{Ne}$ laser for an even simpler experimental configuration, since focusing would not then be required.

We observe that the use of a two-quantum photomixer in a three-frequency nonlinear heterodyne detection receiver [45] would result in a reduction of the SNR by the factor $\left(P_{1} / P_{2}\right)$, corresponding to the absorption of 2 nonmonochromatic photons as discussed earlier. It therefore does not appear to be suitable for this application. We also note that information relating to the intermediate-state lifetime of the detector $\left(\tau_{l}\right)$ can be obtained by measuring the two-quantum detector output for various values of $\tau_{c}$.

Although the emphasis in this paper has been on linearly polarized incident radiation, considerable enhancement of 
the $k$-quantum photocurrent may occur for circularly (or elliptically) polarized radiation, as recently discussed by a number of authors [46].

\section{ACKNOWLEDGMENT}

The author wishes to thank Prof. G. J. Wolga of Cornell University for many valuable discussions in connection with the experiments reported here.

\section{REFERENCES}

[1] A. Einstein, "Concerning a heuristic point of view toward the emission and transformation of light," Ann. Physik., vol. 17, p. 132, 1905 (transl.: Amer. J. Phys., vol. 33, pp. 367-374, May 1965).

[2] a) L. Mandel, "Fluctuations of photon beams: The distribution of the photo-electrons," Proc. Phys. Soc. (London), vol. 74, pp. 233-243, Sept. 1959.

b) See also E. M. Purcell, "The question of the correlation between photons in coherent rays," Nature, vol. 178, pp. 14491450, Dec. 1956.

c) L. Mandel, "Fluctuations of photon beams and their correlations," Proc. Phys. Soc. (London), vol. 72, pp. 1037-1048, Dec. 1958.

[3] a) R. J. Glauber, "The quantum theory of optical coherence," Phys. Rev., vol. 130, pp. 2529-2539, June 1963.

b) - "Coherent and incoherent states of the radiation field," Phys. Rev., vol. 131, pp. 2766-2788, Sept. 1963.

[4] P. L. Kelley and W. H. Kleiner, "Theory of electromagnetic field measurement and photoelectron counting," Phys. Rev., vol. 136, pp. A316-A334, Oct. 1964.

[5] M. C. Teich and G. J. Wolga, "Work-function estimation with a single yield measurement,"J. Opt. Soc. Amer., vol. 57, pp. 542543, Apr. 1967.

[6] M. C. Teich, J. M. Schroeer, and G. J. Wolga, "Double-quantum photoelectric emission from sodium metal," Phys. Rev. Lett., vol. 13, pp. 611-614, Nov. 1964.

[7] H. Sonnenberg, H. Heffner, and W. Spicer, "Two-photon photoelectric effect in $\mathrm{Cs}_{3} \mathrm{Sb}$," Appl. Phys. Lett., vol. 5, pp. 95-96, Sept. 1964.

[8] M. C. Teich, "Two quantum photoemission and dc photomixing in sodium," Ph.D. dissertation, Cornell University, Ithaca, N.Y., Feb. 1966, unpublished (also Tech. Rep. 453, Materials Science Center, Cornell University, Ithaca, N.Y., Feb. 1966).

[9] M. C. Teich and G. J. Wolga, "Two-quantum volume photoelectric effect in sodium," Phys. Rev., vol. 171, pp. 809-814, July 1968.

[10] F. Shiga and S. Imamura, "Experiment on relation between twophoton absorption and coherence of light," Phys. Lett., vol. 25A, pp. 706-707, Nov. 1967.

[11] E. M. Logothetis and P. L. Hartman, "Three-photon photoelectric effect in gold," Phys. Rev. Lett., vol. 18, pp. 581-583, Apr. 1967.

[12] - "Laser-induced electron emission from solids: Many-photon photoelectric effects and thermionic emission," Phys. Rev., vol. 187 , pp. $460-474$, Nov. 1969.

[13] R. E. B. Makinson and M. J. Buckingham, "The second order photoelectric effect at a metal surface," Proc. Phys. Soc. A (London), vol. 64, pp. 135-139, Feb. 1951.

[14] R. L. Smith, "Two-photon photoelectric effect," Phys. Rev., vol. 128, pp. 2225-2229, Dec. 1962.

[15] H. C. Bowers, "Theoretical and experimental considerations of the double-quantum photoelectric effect," M. S. thesis, Cornell University, Ithaca, N.Y., Feb. 1964, unpublished.

[16] 1. Adawi, "Theory of the surface photoelectric effect for one and two photons," Phys. Rev., vol. 134, pp. A788-A798, May 1964.

[17] P. Bloch, "Preliminary investigation of the two-photon photoelectric effect," J. Appl. Phys., vol. 35, pp. 2052-2054, July 1964.

[18] M. C. Teich and G. J. Wolga, "Multiple-photon processes and higher order correlation functions," Phys. Rev. Lett., vol. 16, pp. 625-628, Apr. 1966.

[19] P. Lambropoulos, C. Kikuchi, and R. K. Osborn, "Coherence and two-photon absorption," Phys. Rev., vol. 144, pp. 1081-1086, Apr. 1966.
[20] B. R. Mollow, "Two-photon absorption and field correlation functions," Phys. Rev., vol. 175, pp. 1555-1563, Nov. 1968.

[21] G. S. Agarwal, "Field-correlation effects in multiphoton absorption processes," Phys. Rev. A, vol. 1, pp. 1445-1459, May 1970.

[22] M. C. Teich, R. L. Abrams, and W. B. Gandrud, "Photon-correlation enhancement of SHG at $10.6 \mu \mathrm{m}$," Opt. Commun., vol. 2, pp. 206-208, Oct. 1970.

[23] P.Diament and M. C. Teich, "Relation between radiation statistics and two-quantum photocurrent spectra," J. Opt. Soc. Amer., vol. 59, p. 661, May 1969.

[24] a) C. Freed and H. A. Haus, "Photocurrent spectrum and photoelectron counts produced by a gas laser," Phys. Rev., vol. 141, pp. 287-298, Jan. 1966.

b) G. Lachs, "Effects of photon bunching on shot noise in photoelectric detection," J. Appl. Phys., vol. 39, pp. 4193-4199, Aug. 1968.

[25] M. C. Teich and P. Diament, "Two-photon counting statistics for laser and chaotic radiation," J. Appl. Phys., vol. 40, pp. 625633, Feb. 1969

[26] P.P. Barashev, "Statistical characteristics of a multiphoton photocurrent," Zh. Eksp. Teor. Fiz., vol. 59, pp. 1318-1326, Oct. 1970 (transl.: Sov. Phys.-JETP, vol. 32, pp. 720-723, Apr. 1971).

[27] - "The multiquantum photoemissive effect in condensed media and statistical characteristics of the multiquantum photocurrent," Phys. Status Solidi (a), vol. 9, pp. 9-43 (pt. I) and pp. 387-414 (pt. II), Jan. and Feb. 1972.

[28] A. T. Forrester, R. A. Gudmundsen, and P. O. Johnson, "Photoelectric mixing of incoherent light," Phys. Rev., vol. 99, pp. 1691-1700, Sept. 1955.

[29] A. Javan, E. A. Ballik, and W. L. Bond, "Frequency characteristics of a continuous-wave He-Ne optical maser," J. Opt. Soc. Amer., vol. 52, pp. 96-98, Jan. 1962.

[30] a) B. J. McMurtry and A. E. Siegman, "Photomixing experiments with ruby optical maser and traveling wave microwave phototubes," Appl. Opt., vol. 1, pp. 51-53, Jan. 1962.

b) A. E. Siegman, S. E. Harris, and B. J. McMurtry, "Optical heterodyning and optical demodulation at microwave frequencies," in Optical Masers, J. Fox, Ed. New York: Wiley, 1963, pp. 511-527.

[31] M. C. Teich, R. J. Keyes, and R. H. Kingston, "Optimum heterodyne detection at $10.6 \mu \mathrm{m}$ in photoconductive $\mathrm{Ge}: \mathrm{Cu}$, , Appl. Phys. Lett., vol. 9, pp. 357-360, Nov. 1966.

[32] M. C. Teich, "Infrared heterodyne detection," Proc. IEEE, vol. 56, pp. 37-46, Jan. 1968 (Reprinted in Infrared Detectors, R. D. Hudson, Jr. and J. W. Hudson, Ed. Stroudsburg: Dowden, Hutchinson and Ross, 1975).

[33] a) B. M. Oliver, "Signal-to-noise ratios in photoelectric mixing," Proc. IRE (Corresp.), vol. 49, pp. 1960-1961, Dec. 1961.

b) H. A. Haus, C. H. Townes, and B. M. Oliver, "Comments on 'Noise in photoelectric mixing,", Proc. IRE (Corresp.), vol. 50, pp. 1544-1546, June 1962.

[34] a) A. E. Siegman, "The antenna properties of optical heterodyne receivers," Proc. IEEE (Special Joint Issue on Optical Electronics), vol. 54, pp. 1350-1356, Oct. 1966.

b) L. Mandel and E. Wolf, "Optimum conditions for heterodyne detection of light," J. Opt. Soc. Amer., vol. 65, pp. 413-420, Apr. 1975.

[35] W. S. Read and D. L. Fried, "Optical heterodyning with noncritical angular alignment," Proc. IEEE (Corresp.), vol. 51, p. 1787, Dec. 1963.

[36] M. C. Teich, "Homodyne detection of infrared radiation from a moving diffuse target," Proc. IEEE, vol. 57, pp. 786-792, May 1969.

[37] M. C. Teich and R. Y. Yen, "On the signal-to-noise ratio for optical heterodyne detection," J. Appl. Phys., vol. 43, pp. 24802481 , May 1972.

[38] a) U. M. Titulaer and R. J. Glauber, "Correlation functions for coherent fields," Phys. Rev., vol. 140, pp. B676-B682, Nov. 1965 .

b) -, "Density operators for coherent fields," Phys. Rev., vol. 145, pp. 1041-1050, May 1966.

[39] M. C. Teich, "Field-theoretical treatment of photomixing," Appl. Phys. Lett., vol. 14, pp. 201-203, Mar. 1969.

[40] - "Quantum theory of heterodyne detection," in Proc. 3rd Photoconductivity Conf., E. M. Pell, Ed. New York: Pergamon, 1971, pp. 1-5.

[41] -, "Coherent detection in the infrared," in Semiconductors and 
Semimetals, R. K. Willardson and A. C. Beer, Ed. New York: Academic, 1970, vol. 5, Infrared Detectors, ch. 9, pp. 361-407.

[42] - "Nonlinear heterodyne detection," in Topics in Applied Physics, R. J. Keyes, Ed. New York: Springer, 1976, Optical and Infrared Detectors, to be published.

[43] a) L. Mandel, "Complex representation of optical fields in coherence theory," J. Opt. Soc. Amer., vol. 57, pp. 613-617, May 1967.

b) L. Mandel and E. Wolf, "Coherence properties of optical fields," Rev. Mod. Phys., vol. 37, pp. 231-287, Apr. 1965.

[44] M. C. Teich, D. A. Berkley, and G. J. Wolga, "Silicon-controlledrectifier long-pulse driver for injection lasers," Rev. Sci. Instr., vol. 36 , pp. 973-974, July 1965 .

[45] a) M. C. Teich, "Three-frequency heterodyne system for acquisi- tion and tracking of radar and communications signals," Appl. Phys. Lett., vol. 15, pp. 420-423, Dec. 1969.

b) M. C. Teich and R. Y. Yen, "Three-frequency nonlinear heterodyne detection. 1: cw radar and analog communications," Appl. Opt., vol. 14, pp. 666-679, Mar. 1975.

c) - "Three-frequency nonlinear heterodyne detection. 2: Digital communications and pulsed radar," Appl. Opt., vol. 14, pp. 680-688, Mar. 1975.

[46] a) R. A. Fox, R. M. Kogan, and E. J. Robinson, "Laser triplequantum photoionization of cesium," Phys. Rev. Lett., vol. 26, pp. 1416-1417, June 1971 .

b) S. Klarsfeld and A. Maquet, "Circular versus linear polarization in multiphoton ionization," Phys. Rev. Lett., vol. 29, pp. 79-81, July 1972.

\title{
Studies of Relaxation Oscillations in Organic Dye Lasers
}

\author{
CHINLON LIN
}

\begin{abstract}
The transient phenomena of relaxation oscillations and "initial spikes" in organic dye lasers are studied experimentally and theoretically. Both damped relaxation oscillations and initial spikes are clearly observed in $\mathrm{N}_{2}$ laser-pumped dye lasers under appropriate conditions which are furthermore shown to agree with theoretical considerations. Linearized rate equations obtained with small-signal approximation are used to describe the initial-spiking phenomenon; for the more general phenomenon of relaxation oscillations computer solutions of the coupled rate equations are obtained. Comparisons with experimental observations show excellent qualitative agreement. Based on the results of these studies, a general technique using the idea of controlled resonator transients is described for tunable subnanosecond pulse generation from laser-pumped dye lasers in the near UV and the visible region of the spectrum.
\end{abstract}

\section{INTRODUCTION}

$\mathrm{T}$ HE PHENOMENON of relaxation oscillations or "spiking" has been observed and studied extensively in solidstate lasers under pulsed excitation [1], [2]. Although in most cases random spiking with pulses of irregular spacings and amplitudes were observed, there were observations of perfectly regular damped relaxation oscillations [2], [3]. Such regular damped relaxation oscillations are expected from theoretical analyses using the coupled rate equations [4]-[6] . It is generally recognized to be a phenomenon due to the interaction between the excess population inversion of the active medium and the photon energy of the electromagnetic field inside the resonator. The characteristics of the relaxation oscillations depend on the rate of change of the population inversion due to pumping, spontaneous decay and stimulated

Manuscript received February 7, 1975.

The author is with Bell Laboratories, Holmdel, N.J. 07733. emission, and the rate of buildup and decay of photons in the resonator due to stimulated emission and various loss mechanisms such as output coupling, scattering, diffraction, and absorption.

In addition to being a common phenomenon in solid-state lasers, relaxation oscillations have also been observed and studied in gas lasers [7]. In the case of dye-solution lasers, relaxation oscillations have not been previously reported. However, in the first organic dye laser, Sorokin et al. [8] noticed that under certain conditions a $Q$-switched ruby laser-pumped dye laser had an "initial spike" in the output, which otherwise displayed the time variation similar to that of the pumping pulse. The observation was shown to agree qualitatively with the expected transient response of the dye laser under the corresponding experimental conditions. Miyazoe and Maeda [9] have further applied small-signal analysis to study the conditions for the appearance of such initial spikes. They pointed out that dye lasers are generally operated close to the border region between spiking and nonspiking. This is in contrast to solid-state lasers for which the spiking phenomenon is almost ubiquitous.

In this paper we report the observation and investigation of regular damped relaxation oscillations in $\mathrm{N}_{2}$ laser-pumped dye lasers. Pulses with an initial spike are also observed; it is shown to be a special case of the more general phenomenon of relaxation oscillations. The characteristics of these transient responses are studied experimentally and compared with theoretical considerations. Excellent qualitative agreement is obtained. Because of the relative ease and large workable range in choosing desired dye-laser parameters (e.g., dye-laser medium and resonator length, resonator mirror reflectivities, dyesolution concentration, dye-fluorescence lifetime, etc.) laser- 